\title{
DESIGN, FABRICATION AND MEASUREMENT OF A TUNNELING TIP ACCELEROMETER
}

\author{
Jianchao Wang, Bob McClelland, and Paul M. Zavracky \\ Northeastern University, Department of Electrical and Computer Engineering \\ 360 Huntington Avenue, Boston, MA 02115 \\ Frank Hartley and Ben Dolgin \\ Jet Propulsion Laboratory \\ 4800 Oak Grove Drive, Pasadena, CA 91190
}

\begin{abstract}
We have designed and fabricated a bulk micromachined single axis accelerometer. The accelerometer is designed to have a full scale range of one milli-g and a sensitivity of $10^{-8} \mathrm{~g}$. This is accomplished by using an ultrahigh sensitive tunneling tip as a displacement sensor. The device consists of four silicon dice which are assembled together using eutectic bonding technique. Each die is processed separately before bonding. The fabrication procedures for the three different dice are described Electrical contacts are made between layers during the bonding operation. The force feedback control approach is used for the accelerometer system. The clamping function for the parking mode is fully tested. The preliminary testing on the tip control function is performed. Initial results of acceleration measurement including the time domain response and power spectra are presented
\end{abstract}

\section{INTRODUCTION}

After the first report of a silicon accelerometer by Roylance and Angell [1] in 1979, many kinds of micromachined silicon accelerometers with different operating principles and structures have been developed. Among these sensors, piezoresistive [2] and capacitive [3, 4] accelerometers are the most common and well developed. These accelerometers have demonstrated good performance with sensitivities down to a micro-g. However, for applications below a micro-g, as in microgravity research in the space shuttle; measurement of orbital drag; and active aiming and isolation systems, the traditional position sensing methods have their limitations and a new position transducer must be used.

In this paper, we describe the development of a micromachined accelerometer which is designed to be capable of measuring $10^{-8} \mathrm{~g}$. Our approach to ultrahigh resolution devices is to incorporate weak springs and a relatively large mass with an ultra-sensitive tunneling tip position detector. Electron tunneling through a vacuum barrier between a tip electrode and a plane electrode has been successfully used in Scanning Tunneling Microscopy (STM)[5] to achieve real space images on an atomic scale. With the development of micromachining technology, tunneling tips have also found applications in sensors with reported resolution as low as hundredths of an Angstrom [6-8]. This is due to the fact that the electron tunneling current is exponentially proportional to the electrode separation. Theoretical analysis has shown that for a typical tunneling spacing of $10 \AA$ and a barrier height of $4 \mathrm{eV}$, the tunneling current increases by one order of magnitude for each $1 \AA$ decrease in electron separation.

We also use a force feedback control in which the position of the proof mass is held constant. This is accomplished by feeding back position information to the control electrodes. Using force feedback can improve the linearity, increase the dynamic range, and stabilize the system. Key elements of our design include the optimized placement of the tip, the minimization of off-axis sensitivity and tip protection for over loading.

\section{DESIGN FEATURES}

An accelerometer usually consists of a proof mass to convert an acceleration to a force, a spring to balance the acceleration force and a position detector to sense the position changed due to acceleration. When the system is experiencing an acceleration, the proof mass will move from its original position to a new position which is determined by the balance between inertial force on the proof mass and the spring force. With a simple mechanical spring, the acceleration will be directly proportional to the distance traversed by the proof mass from its equilibrium position. In order to build an micro-gravity accelerometer, a large proof mass, weak springs and an ultra-sensitive position detector are needed. In this design, the use of an electron tunneling tip is fundamental to its superior performance. The basic design features and specifications for the accelerometer are listed in Table 1 and Table 2, respectively.

Table 1

Design Features

\begin{tabular}{l} 
Electron Tunneling Tip Position Detection \\
Electrostatic Force Feedback \\
Pitch and Roll Control \\
High Off-axis Insensitivity \\
\hline
\end{tabular}

Table 2 Specifications

Parameter

Specification

\begin{tabular}{lc}
\hline Full-Scale Sensitivity & $10 \mathrm{mg}$ \\
Resolution & $1 \times 10^{-8} \mathrm{~g}$ \\
Off-Axis Sensitivity & $1 \times 10^{-9} \mathrm{FS}$ \\
Bandwidth & $20 \mathrm{~Hz}$ \\
Built in Tunneling Tip Protection & $>32 \mathrm{~g}$ \\
\hline
\end{tabular}

The required proof mass is achieved by producing a relatively large $\left(-2 \mathrm{~cm}^{2}\right)$ bulk micromachined device in two separate wafers that are eutectically bonded together. The proof mass is held to the surrounding frame by a set of springs referred to as 'crab legs' [9]. These weak springs are fabricated by etching back the silicon to form $25 \mu \mathrm{m}$ thick diaphragms which are further patterned to form $2 \mathrm{~cm}$ long folded spring elements. The resulting spring constant is only $1.7 \mathrm{~N} / \mathrm{m}$. Since the tunneling tip must be held in near proximity (10 $\AA$ ) to the proof mass during operation, electrostatic force feedback has been employed. This is coupled to the proof mass on one side through a matrix of four plates that surround the tunneling tip and a single force plate on the other. 
An important design issue is the minimization of off-axis sensitivity. With the unique design of the crab leg springs, calculation shows that the lateral spring constant is nearly five orders of magnitude higher than the normal spring constant. Further minimization of the off-axis sensitivity can be accomplished by ensuring that the proof mass and spring design is symmetric, and the spacing between the neutral position of the proof mass and the top of the tip is zero. As a consequence, thermal sensitivity is also reduced. To further reduce thermal sensitivity, all dice used in the device are fabricated in silicon. This choice minimizes stresses that can arise from thermal mismatch. Another issue considered in our design is that of tip protection. The single force plate doubles as a parking electrode designed to attract and hold the proof mass in place using a low voltage $(<15$ V).

\section{FABRICATION}

The accelerometer [10] is fabricated using bulk micromachining technology. The cross sectional view of the finished device is shown in Fig.1. It is constructed from four dice, with the proof mass assembled from two identical dice. Three different processes for the proof mass, force plate and tip plate are described below.

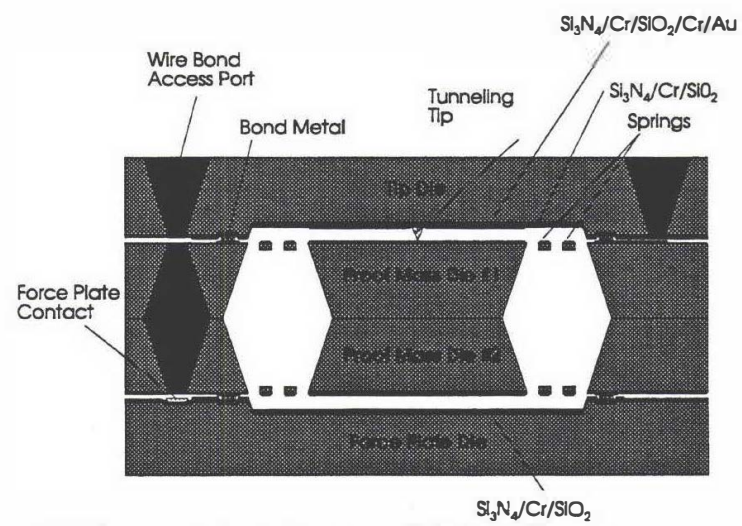

Figure 1. Cross-section of an assembled accelerometer.

The proof mass wafers (Fig. 2) are back etched in $25 \% \mathrm{KOH}$ to define the proof mass and separation channel. At the same time, vias are etched to permit wire bonding through the assembled structure. The back side etch continues until the remaining diaphragm is 25 microns thick. This is a timed etch and no dopant stop is used in order to avoid strain related problems. The wafer is stripped back to silicon and recoated with silicon nitride. TiW/Au is deposited on the front side and pattemed appropriately. This metallization provides the electrical contacts to the surface of the proof mass. Additional electrical leads are used to align and route electrical connections through the structure. The wafers are repatterned to the spring mask and etched anisotropically in a plasma etcher. This deep plasma etch releases both the springs and the dice. Two proof mass dice are then cleaned and bonded together eutectically.

The force plate wafer is deep etched in a manner similar to the proof masses to define the separation channel. Each wafer contains a grounded bond ring that surrounds the central well and is electrically isolated from the other electrical features. The force plate wafer contains a single $5 \mu \mathrm{m}$ deep well in the center of the die. A two metal system is employed. The first metal is Chromium which is deposited by sputtering and patterned lithographically. It is then covered with an LTO layer which is patterned to open vias to the first metal. The second metal is TiW/Au which is patterned to form the bond regions and the wire bond pads. When completed, the force plate die is bonded to one of the assembled proof mass die in such a way that the side of the proof mass die with the springs is the side that is bonded.
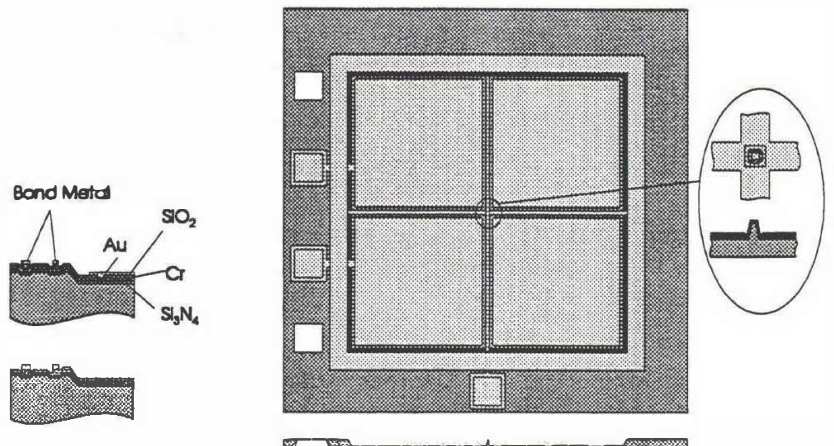

Figure 2. Various views of the tip die.

The tip dice (Fig. 2) undergo a similar initial process. The backside is deep etched in $\mathrm{KOH}$ for die separation purposes. The

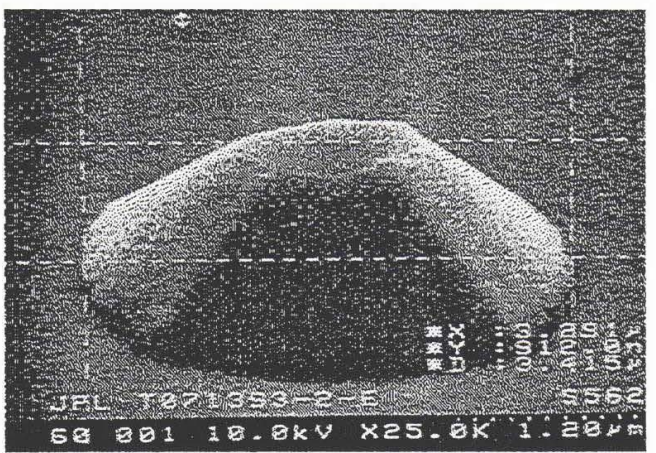

Figure 3. An SEM micrograph of a flat tunneling tip.

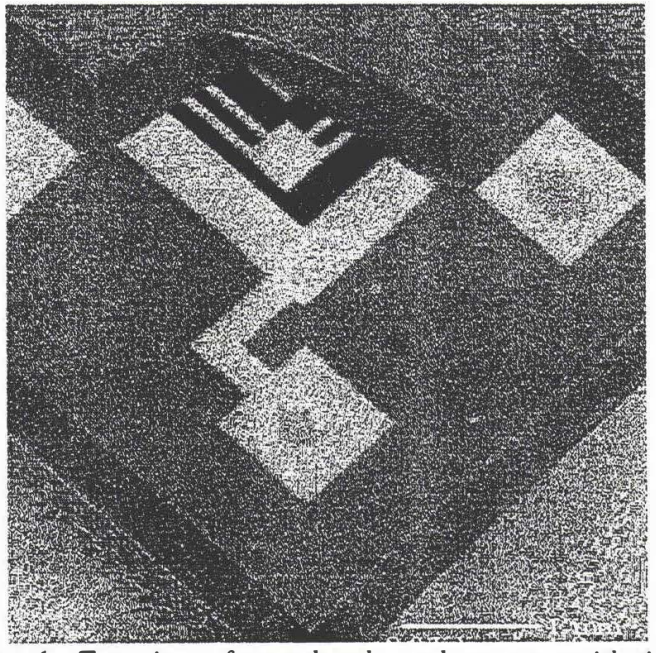

Figure 4. Top view of completed accelerometer with tip die cut away to reveal the internal structure using a SEM.

tip is created by a sequence of dry and four anisotropic wet etch steps which result in a flat topped tip. The tip is surrounded by four electrodes and sits in a well. Grooves are also provided for a eutectic bond ring. Again, a two metal process similar to that of the force plate is used. Electrical leads to the force plates and tip 
connect to contact pads at the periphery of the die. A final plasma etch process is used to separate individual die. The completed tip die is eutectically bonded to the proof mass. Since the tip die is placed above the proof mass during assembly, the proof mass is held away from the tip by gravity. This prevents damage to the tip during bonding. The SEM pictures for the tunneling tip and part of the completed accelerometer are shown in Fig. 3, and Fig. 4, respectively.

\section{EXPERIMENTAL RESULTS}

The accelerometer has four modes of operation: parking, transition to operation mode under capacitance control, operational mode (tunneling control), and overload protection. The device has been fully tested in the parking mode. Tunneling tip control has also been tested.

Parking of the proof mass protects the fragile structures (tunneling tip and the flexures) from damage during shipment, handling, and spacecraft launch. The ability of the parking mechanism to withstand the high load was tested by applying a large force directly to a parked proof mass (the quadrant plate was removed). Fig. 5 shows the value of the force required to dislodge the proof mass as a function of the applied voltage. The force is expressed in units of acceleration of the proof mass $(F=m a)$. The theoretical curves correspond to zero and $1 \mu \mathrm{m}$ average separation between the proof mass and the force plate. No stiction of the proof mass was observed after parking for as long as four days.

The feasibility of the tunneling tip control was tested by using a simple control circuit in a $1 \mathrm{~g}$ field. The block diagram for the control system is shown in Fig.6. The closed-loop control system is linearized at the operating point where the tunneling spacing is $10 \AA$. It should be noted that the bias voltage, $V$, used to

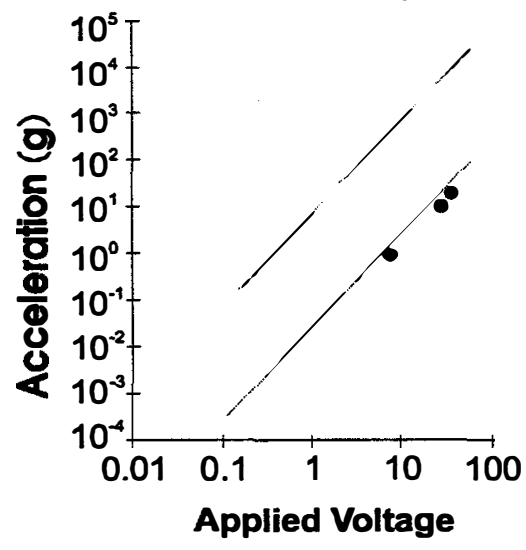

Figure 5. Experimental and theoretical data showing the maximum achievable acceleration against which the force plate can hold as a function of the voltage difference between the force plate and proof mass.

offset the background $1 \mathrm{~g}$ gravitational field, influences the magnitude of the effective spring constant for the mechanical system and the return ratio for the system. It can be shown that the system is stable as long as the damping term for the accelerometer is large. This is easily accomplished by squeeze film damping when operating at one atmosphere. A circuit board was fabricated and included a special zero insertion force connector for the packaged accelerometer and the control circuit. The unit was slowly turned to a position where the proof mass is resting on the tunneling tip. At this point, the tunneling current electronics would register a high current. The feedback loop was turned on next. A noise spectrum as shown in Fig. 7 is obtained using a HP dynamic signal analyzer with the device operated at

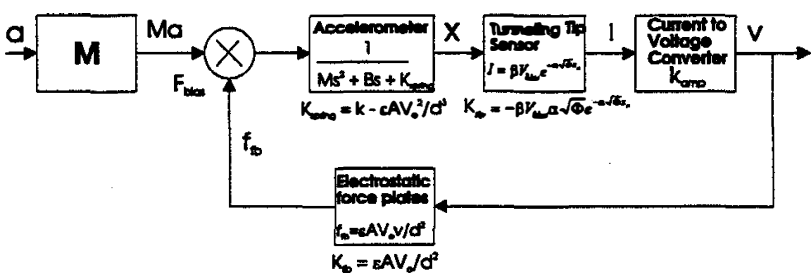

Figure 6. A block diagram of the force feedback control system used to measure the tunneling tip accelerometer.

Accelerometer Noise Spectrum

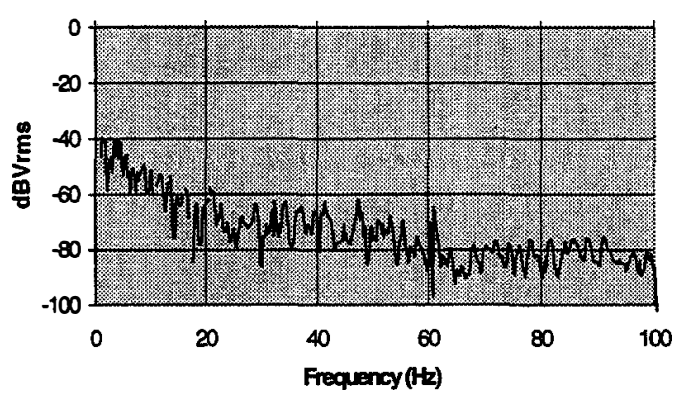

Figure 7. Accelerometer noise spectrum for a frequency range from $1 \mathrm{~Hz}$ to $100 \mathrm{~Hz}$.

atmosphere. It was found that the flicker noise dominates the low frequency region, where the highest value corresponds to 2 milli$\mathrm{g} / \mathrm{Hz}^{1 / 2}$. Comparing the result from the reference accelerometer and the noise from the circuit, we believe that this $1 /$ f noise comes from the electron tunneling process. It may result from the migration of atoms on the active electrode surface, surface diffusion of adsorbed molecules and the adsorption-desorption process[11].

The acceleration response of the tunneling tip accelerometer was tested on a vibration isolation stage. The stage is constructed using a $100 \mathrm{lb}$ platform suspended by 646 -feet long bungee cords. The tunneling tip accelerometer is mounted on a thrust stand sitting on the platform. A reference accelerometer (Sunstrand QA3000) with sub- $\mu$ g sensitivity was also installed on the stand for calibration purpose. The motion of the unit including both accelerometers was controlled by a piezo-driver which was able to generate sinusoidal accelerations at frequencies from $\mathrm{DC}$ to about $200 \mathrm{~Hz}$. Fig. 9 shows the responses of both the tunneling tip accelerometer and the Sunstrand QA3000 at $53 \mathrm{~Hz}$ with an amplitude of $80 \mathrm{mg}$. Data from the QA3000 was collected with a 2nd order Butterworth filter during the measurement whereas the data for the tunneling tip device was unfiltered. The data for the tunneling tip device shows a higher frequency $(628 \mathrm{~Hz})$ signal overlaid on the $53 \mathrm{~Hz}$ response. This may be a resonance mode of the spring system. For the measurement at much lower frequencies, the tunneling tip accelerometer is put into a vacuum holder in order to keep the device clean and reduce the noise. The bandwidth of the measurement system is set to $10 \mathrm{~Hz}$. The data is then digitized and converted to power spectra using Fast Fourier transform (FFT) techniques. One of the results is shown in Fig. 9, where the exciting frequency is $0.2 \mathrm{~Hz}$ and the magnitude is $1 \mathrm{mg}$. In order to obtain the power spectrum for a $0.2 \mathrm{~Hz}$ signal, the measurement time would be at least 8 minutes. It can be seen that the two responses from the tunneling tip accelerometer and the QA 3000 are consistent in the vicinity of the highest peak $(0.2 \mathrm{~Hz})$. 


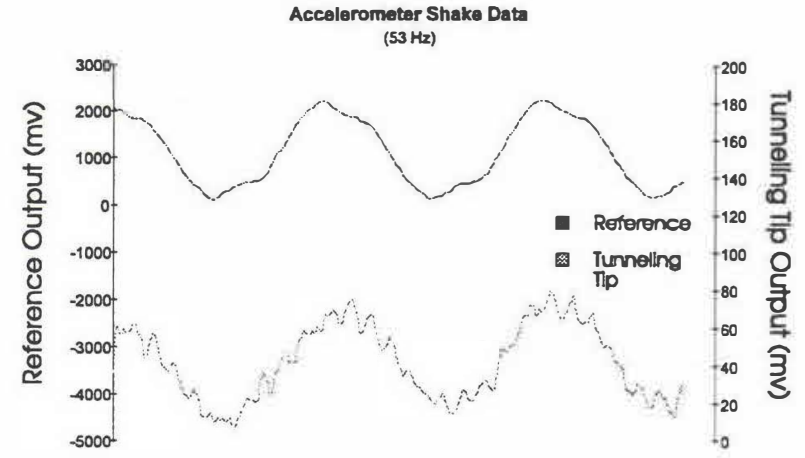

Figure 8. Accelerometer data comparing a Sunstrand QA3000 (reference) with the tunneling tip accelerometer.

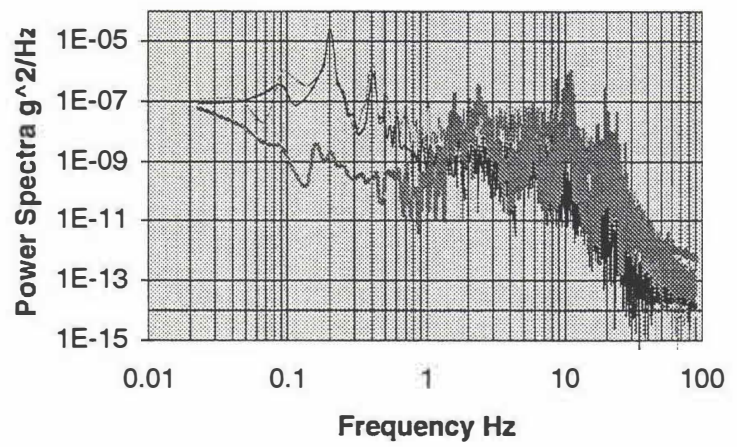

Figure 9. Power spectra for the responses to a $0.2 \mathrm{~Hz} 1 \mathrm{mg}$ excitation. The dark one of the upper two curves is the response of the tunneling tip accelerometer. The light one is from QA3000. The lowest curve is the background noise.

\section{CONCLUSIONS}

We have reported the design considerations, fabrication procedures and some experimental results for an ultra-sensitive accelerometer. The accelerometer is fabricated using a bulk micromachining approach. Three distinctly different dice are processed separately and then eutecticly bonded together to form the final device. The bonding process also provides hermetic sealing and electrical interconnection between dice. The accelerometer is designed with a force plate that can be used to hold the proof mass and spring assembly electrostatically during transportation. The capability of the electrostatic clamping mechanism has been demonstrated. A linear closed-loop control model for the force feedback accelerometer system is presented in a block diagram. From preliminary measurement, we found that the device suffers from $1 /$ f noise for operations in atmosphere. The response to a sinusoidal excitation in time domain is demonstrated and compared with a calibration accelerometer. The acceleration measurement at very low frequencies was performed. The resulted power spectrum shows good agreement between the tunneling tip accelerometer and the reference accelerometer in the vicinity of the signal frequency.

\section{ACKNOWLEDGMENTS}

The authors would like to acknowledge the help and support of JPL. We also appreciate the assistance of Keith Warner in reviewing this document.

\section{REFERENCES}

1. L. M. Roylance and J. B. Angell, "A Batch-Fabricated silicon accelerometer”, IEEE Trans. Electron Devices, Vol. ED-26, No. 12 December 1979, pp. 1911-1917.

2. H. Seidel, U. Fritsch, R. Gottinger, J. Schalk, J. Walter and K. Ambaum, "A Piezoresistive Silicon Accelerometer with Monolithically Integrated CMOS-Circuitry", The 8th Int. Conf. on Solid-State Sensors and Actuators, and Eurosensors IX, Stockholm, Sweden, pp. 150-C4, June 25-29, 1995.

3. F. Rudolf, A. Jornod, J. Bergqvist and H. Leuthold, "Precision Accelerometers With $\mu \mathrm{g}$ Resolution", Sensors and Actuators, A 21-A23, 1990, pp. 297-302.

4. E. Peeters, S. Vergote, B. Puers and W. Sansen, "A highly symmetrical Capacitive Micro-Accelerometer With Single Degree of Freedom Response", J. Micromech. Microeng. Vol. 2, pp. 104$112,1992$.

5. Y. Kulk and P. J. Silverman, "Scanning Tunneling Microscope Instrumentation", Rev. Sci. Instrum. 60(2), February 1989, pp. 165-180.

6. T. W. Kenny, W. J. Kaiser, H. K. Rockstadm J. K. Reynold, J. A. Podosek, and E. C. Vote, "Wide-Bandwidth Electromechanical Actuators for Tunneling Displacement Transducers", J. MEMS. Vol. 3, No. 3, pp. 97-104, September 1994.

7. T. W. Kenny, W. J. Kaiser, S. B. Waltman, and J. K. Reynold, "A Novel Infrared Detector Based on a Tunneling Displacement Transducer”, Appl. Phys. Lett, Vol. 59, pp. 1820-1822, 1991.

8. H. K. Rockstad, J. K. Reynold, T. K. Tang, T. W. Kenny, W. J. Kaiser, and T. B. Gabrielson, "A miniature, high-sensitivity, electron tunneling accelerometer", Transducers'95, pp407 (PC 12).

9. P. M. Zavracky, F. Hartley, and D. Atkins, "New Spring Design and Processes for an Electron Tunneling Tip Accelerometer", to be published.

10. P. M. Zavracky, F. Hartley, N. Sherman, T. Hansen, and K. Wamer, "A New Force Balanced Accelerometer Using Tunneling Tip Position Sensing", 7th Int. Conf. on Sensors and Actuators, Yokahama, Japan, June 7-10, 1993, pp. 50-51.

11. T. Tiedje, J. Varon, H. Deckman and J. Stokes, "Tip Contamination Effects in Ambient Pressure Scanning Tunneling Microscopy Imaging of Graphite”, J. Vac. Sci. Technol. A 6(2), Mar/Apr., 1988, pp. 372. 